\title{
CÓDIGO TÉCNICO Y SITUACIÓN HERMENÉUTICA
}

\section{TECHNICAL CODE AND HERMENEUTIC SITUATION}

\author{
LUCIANO MASCARÓ* \\ CONICET, ANCBA-CEF
}

Resumen: En su obra transforming technology, Andrew Feenberg presenta a Heidegger como representante de la así llamada "teoría sustantiva de la tecnología». En el presente trabajo buscamos mostrar 1) que dicha interpretación responde al hecho de que el autor canadiense circunscribe su análisis casi exclusivamente a las obras del segundo período del pensar de M. Heidegger, y 2) que, de haber centrado su estudio en las obras de anteriores a los años '30, Feenberg hubiera encontrado en el autor alemán, un antecedente para su sugerida "teoría crítica de la tecnología». En esta línea, nos ocuparemos de hacer explícita una de las posibles articulaciones de ambos pensamientos: propondremos que, en la noción de "Código técnico» de A. Feenberg, puede encontrarse una fuerte resonancia de la idea de Mundo de Heidegger.

Palabras clave: Código Técnico, Mundo, Situación hermenéutica, Heidegger, Feenberg, Técnica

"Doctor en Filosofía por la Universidad de Buenos Aires. Profesor Adjunto en la materia Metafísica, en la carrera de Filosofía de la Universidad de Ciencias Empresariales y Sociales (UCES). Profesor adjunto en la materia Filosofía del lenguaje, en la carrera de Filosofía de la Universidad Católica Argentina (UCA).

Contacto: lcnmascaro@hotmail.com Calle Soler 5565 1B, Capital Federal, Buenos Aires, Argentina. El manuscrito ha sido elaborado gracias a la subvención de la beca postdoctoral del Consejo Nacional de Investigaciones Científicas y Técnicas (CONICET), lugar de trabajo: Academia Nacional de Ciencias de Buenos Aires, Centro de Estudios Filosóficos (ANCBA-CEF) 
Aвstract: The following research attempts to study Andrew Feenberg's work "Transforming Technology" in order to show that the interpretation of Heidegger as a representative of the so-called "Substantive theory of technology" responds to the selective reading of works from the period after the 1930's "Kehre." In contrast, we hold that Heidegger's philosophy from the 1920's has numerous contact points with Feenberg's own position, the so-called "Critical Theory of Technology.» From this perspective, we will seek to make one of the possible linkages explicit: we shall propose that, in Feenberg's notion of "Technical Code,» a strong resemblance with Heideggers concept of «World» can be found. By these means, we try to uphold the German author's figure, as that of a defender, or at least, a relevant background author, for the Critical Theory of Technology.

KeYwords: technical code, world, hermeneutic situation, Heidegger, Feenberg, technology

\section{Introducción}

El presente trabajo persigue dos objetivos: 1) En su obra de 2002 «Transforming Technology», Andrew Feenberg propone una división de las posturas filosóficas en torno a la cuestión de la tecnología: posición sustantiva, instrumental y crítica. El autor canadiense presenta a Heidegger como representante de la primera postura. En el presente trabajo procuraremos mostrar que la lectura sustantivista de la obra de Heidegger responde a un específico recorte de su obra, a saber, la preferente consideración de las obras posteriores a la «Kehre» de los años '30. En contraste, consideramos que, de haberse centrado en la filosofía heideggeriana de los años '20, Feenberg hubiese reconocido en Heidegger un antecedente de la así llamada "teoría crítica de la tecnología». Nos ocuparemos de explicitar los puntos de contacto. 2) En lo particular, nos proponemos mostrar la posible articulación entre una noción central del texto de Feenberg y una idea fundamental en el proyecto de Ser y Tiempo: propondremos que la noción de "código técnico» de Andrew Feenberg es heredera de la idea de Mundo de Heidegger. Por esta vía buscamos rescatar la figura del autor alemán como la de un defensor, o al menos, un antecedente de la interpretación de la realidad técnica propuesta por Feenberg. 


\section{Las tres interpretaciones de la tecnología}

En su obra de 2002 «transforming technology», Andrew Feenberg realiza una distinción inicial en torno a la cual organiza su escrito: él diferencia tres posibles abordajes filosóficos sobre el problema de la neutralidad de la tecnología.

En primer lugar, se menciona la «teoría instrumental» de la tecnología. Esta postura considera que la tecnología es neutral de suyo, y que resulta subordinada a valores de otras esferas. No habría ninguna implicancia política en el diseño tecnológico, y el sesgo social y antropológico del que se revista cada dispositivo vendría dado únicamente por la intención del agente, y los fines a los cuales se oriente la acción.

La segunda postura es la así llamada «teoría sustantiva». Ésta considera que la tecnología posee un sesgo que no sólo le es inherente, sino incluso inmodificable. Según esta postura, la tecnología haría del mundo social un objeto de control. La instrumentalización del mundo es un destino, un ominoso panorama ante el cual se propone un regreso a la tradición y simplicidad, o bien, un abandono ante el horizonte distópico. Feenberg menciona a Heidegger y a Ellul como los dos grandes representantes de esta posición.

La tercera posición -la variante interpretativa propuesta por Feenberg- se ubica, en cierto modo, en medio de las dos anteriores. Efectivamente, la así llamada "teoría crítica de la tecnología» no cae en la consideración simplista de la postura instrumental; por el contrario, se reconoce una fuerte incidencia política así como una carga social y valorativa inherente a los objetos técnicos (no sólo en la fase de diseño, sino también encarnada en el artefacto concreto y en su puesta en práctica) la dimensión valorativa de los objetos técnicos estaría dada, según veremos, por el Código técnico del cual ellos emanan, en el cual se instalan y obtienen su sentido. Por otra parte, la teoría critica de la tecnología tampoco se identifica con la posición «extremista» de la interpretación sustantiva; en efecto, si bien la tecnología está imbuida de un sesgo político y simbólico, ella no representa un «destino» invariable, ante el cual sólo podemos resignarnos, o escapar refugiándonos en la tradición, sino un fenómeno ambivalente (Cfr. Feenberg, 2012:91). La postura crítica considera que el sesgo de la tecnología proviene del código técnico que le da origen, de modo que ningún dispositivo resulta inherentemente represivo o subversivo. La tecnología posee potencialidades (Feenberg, 2012:64), que abren los caminos tanto para un desarrollo 
democrático como para un ejercicio de dominación desde arriba ${ }^{1}$. Incluso más, la teoría crítica considera que la técnica no es sólo un medio, sino un complejo entorno del mundo de la vida (Cfr. Feenberg, 2012:28).

Efectivamente, en la filosofía del segundo Heidegger encontramos una interpretación de la técnica que la convierte en la última etapa en la historia del olvido del ser. Se trata del desarrollo culminante del pensar metafísico, un modo de descubrimiento que hace del mundo un bien en stock, y que sólo lo abre en sus potencialidades utilitarias. Este modo del descubrimiento va acompañado por una actitud de provocación de la naturaleza, que la ubica bajo el dominio del hombre al tiempo que la interpreta como mero depósito de energías.

La posición de Feenberg es diferente, como dijimos, a la del segundo Heidegger, sin embargo, consideramos que si su escrito se hubiese concentrado en las reflexiones de la filosofía del Heidegger de los años '20 (período de Marburgo), muchos puntos de contacto entre ambas filosofías se hubiesen hecho manifiestas, y el canadiense hubiese colocado al autor de Ser y Tiempo en una posición mucho más cercana a la de la teoría crítica de la tecnología. A continuación exploraremos sólo uno de estos posibles puntos de contacto.

\section{La noción de código técnico}

El código técnico -también llamdo "código social de la tecnología»-consiste en un conjunto de regularidades en el diseño de las tecnologías que funciona como condición de realización de un interés en una solución coherente. Este trasfondo para la decisión se vuelve paradigma de un dominio completo de actividad técnica. En el caso del capitalismo, el código técnico favorece la dominación «desde arriba», por ello afirma Feenberg: «el código técnico es una regla según la cual se hacen elecciones técnicas con el fin de preservar la autonomía operacional [del poder hegemónico]» (Feenberg, 2012:127). Este concepto presupone que hay muchas soluciones diferentes para los problemas técnicos, e incluso, más, sugiere que los problemas técnicos son problemas sociales. Por ello puede indicarse que las tecnologías son más que la sumatoria de sus partes; en el diseño, construcción

\footnotetext{
${ }^{1}$ Desde luego, la adaptación de un objeto técnico para amplificar el margen de maniobra de los oprimidos dependerá de un cierto rediseño del artefacto desde el mismo interior del lugar de trabajo
} 
e implementación de un dispositivo, hay una «malla de determinaciones sociales» (Feenberg, 2012:129), que pre-construye un dominio de actividad social.

El código técnico, es, entonces, la intersección de lo social y lo tecnológico, el escenario en que los aspectos duales poder/conocimiento se reconcilian en el objeto técnico; en otras palabras, es el punto en el cual el artefacto se vuelve político.

Feenberg también se refiere al código técnico en estos términos: «es el principio de identidad organizacional y supervivencia» (Feenberg, 2012:127) Esto significa que un sistema técnico (opresivo o no) se sostiene y reproduce codificándose, y retirándose hacia el trasfondo, es decir, volviéndose invisible: el código no es explícito, ni está manifiesto en ningún libro de reglas; justamente en este carácter implícito radica su poder rector de las decisiones cotidianas, políticas, y de diseño.

Este código es el que dictamina todas las particularidades de la escena en la que un objeto técnico hace su aparición. El código predefine ${ }^{2}$ los materiales de los que será hecho el objeto (según la disponibilidad, el costo, la adecuación a la tarea; pero también según la demanda y apreciación estética, o la peligrosidad para la vida humana $)^{3}$; también predefine lo que debe ser construido, lo relevante e irrelevante, lo urgente y lo postergable ${ }^{4}$; a su vez, el código establece (de manera no-manifiesta para diseñadores y usuarios) una serie de valores simbólicos

\footnotetext{
${ }^{2}$ Este carácter previo de la decisión será fundamental a la hora de articular la filosofía de Feenberg con la del primer Heidegger. La de Heidegger es también una filosofía de las potencialidades de la técnica.

${ }^{3}$ Podemos imaginar utensilios, o partes de máquinas hechos de plata, marfil o diamante; de acero o madera, e incluso de materiales cancerígenos como asbesto o plomo.

${ }^{4}$ Pensemos en algunos casos: a) La economía de la Grecia antigua dependía en gran medida de la mano de obra proveniente del trabajo esclavo. Ante esta configuración histórica de la sociedad, se volvía innecesaria la búsqueda de herramientas que reemplazasen el trabajo físico por trabajo mecánico.

b) la lata de conserva fue inventada en 1810, sin embargo, el abrelatas no hace su aparición hasta 1858. Hasta entonces la lata -utilizada en gran medida por soldados y exploradores- era abierta con un cuchillo, una bayoneta, o incluso un disparo de fusil. No fue sino hasta la introducción de latas más livianas y manejables, hechas de acero delgado -hasta entonces, el envase poseía gruesas paredes y llegaba a pesar incluso más que los alimentos que contenía-, y el ingreso de estos recipientes en el ámbito doméstico que se volvió necesaria (y viable) la construcción de un artefacto que simplificase el proceso de apertura.
} 
que serán perseguidos en el diseño 5 . Pero no únicamente estos factores vienen definidos de antemano por el código técnico, también, e incluso de manera más fundamental y relevante, define lo que será un usuario, un trabajador, o un sujeto en general.

Según afirma Feenberg, la herramienta encierra también potencialidades de configuración de los usuarios, "lo que significa ser humano se decide en gran medida en función de la forma de nuestras herramientas» (Feenberg, 2012: 44) Esto significa que el sujeto se configura por las posibilidades que los medios técnicos permiten y se constituye en actor en virtud de esos medios. ¿Podría existir un carpintero sin martillo y serrucho? Pero, desde luego, no será igual el sujeto que quede modalizado por el uso de una sierra doble mango, que el que utilice un serrucho simple; su relación con el mundo, con el trabajo y con los otros se verá modificada; sus reflejos, e incluso los callos de sus manos tomarán diferente forma. Todos estos aspectos se reúnen en lo que Feenberg denomina «instrumentalización secundaria» (Feenberg, 2012:273), esto es, la forma concreta que adquiere un artefacto en un determinado contexto social, y las consecuentes modalizaciones de los usuarios cuyo quehacer se ve definido por dicho útil. El diseño técnico así visto va mucho más allá de la simplista explicación que hace surgir al artefacto de la aplicación de una finalidad a un material disponible. Una teoría de la instrumentalización secundaria nos diría, en palabras de Feenberg, "qué pasa con las personas cuyas vidas están dedicadas a trabajar la madera» (Feenberg, 2012:273). No es solamente porque un dispositivo funciona que se lo elige para ser desarrollado entre otras muchas configuraciones coherentes de elementos técnicos. En numerosas oportunidades, son las condiciones contextuales las que obstaculizan o fomentan el surgimiento de un dispositivo tecnológico ${ }^{6}$.

\footnotetext{
${ }^{5}$ Automóviles de gran tamaño, pesados, angulosos y con (inservibles) alerones en los '50; figuras minimalistas y de colores sobrios en los artículos de informática y telefonía de nuestros tiempos. Cada uno, a su manera y en su época, un símbolo de status y posición social. ${ }^{6}$ pensemos, por ejemplo, en el dilema ético inherente a la patentabilidad de semillas alteradas genéticamente. La misma situación se observa si consideramos un procedimiento como la introducción de un canario en una jaula para detectar niveles nocivos de sustancias químicas en el ambiente; semejante práctica sería imposible, o al menos, ampliamente polémica hoy en día. En este mismo sentido, observamos que la Eolípila de Herón, considerada por muchos la primera máquina de vapor de la historia de occidente, encerraba ya las posibilidades de una revolución industrial, sin embargo, este artefacto no fue más allá de su utilización recreativa como un juguete. ¿por qué los griegos no explotaron las potencialidades de esta nueva forma de generar energía y movimiento mecánico? La respuesta puede encontrarse en la misma estructuración técnica de la sociedad en torno a la fuerza de trabajo esclavo, y la vasta preeminencia del trabajo artesanal y doméstico
} 
Feenberg afirma de manera esclarecedora: «el carácter social de la tecnología descansa no en la lógica de su funcionamiento interno, sino en la relación de esa lógica con un contexto social» (Feenberg, 2012:130) Ciertamente, el craneómetro de Ellis y Grey sólo puede surgir en un contexto donde la frenología se encontraba entre las disciplinas científicas socialmente aceptadas (Cfr. Bud y Warner, 1998:157)

En efecto, lo que define el modo de aparición de un artefacto concreto es, sin dudas, el código técnico (escenario de politización del artefacto), que determina tanto la forma de racionalidad que descubre al mundo (instrumentalización primaria) como la composición y estructuración misma del útil concreto, y sus consecuencias sobre el usuario en tanto agente socialmente localizado (instrumentalización secundaria). Por todo lo dicho, el código técnico pude ser pensado como un control de la significación (Feenberg, 2012:127), o bien, aproximándonos ya a la terminología heideggeriana, un control del horizonte de comprensibilidad del útil. Este complejo horizonte (en el cual la eficiencia es sólo uno de los múltiples factores condicionantes) es el que posibilita el surgimiento de un artefacto en una sociedad concreta.

\section{La filosofía de la praxis del primer Heidegger}

En discusión con la concepción sustantiva, Feenberg afirma que la tecnología no es un destino, sino una "escena de lucha» (Feenberg, 2012:38). Nos preguntamos, pues, ¿qué puede significar aquí «escena»? Escena significa, en el contexto del estudio de Feenberg, horizonte, trasfondo que posibilita la aparición de las cosas, en otras palabras, «mundo» en el sentido elaborado por el primer Heidegger. Esta «escena» es descripta como una escena "de lucha». Aquello que combate sobre este trasfondo son todas las dimensiones de incidencia implicadas en la noción de código técnico, a saber, cargas simbólicas, valores morales y llanamente utilitarios (tales como la noción de eficiencia o productividad), la adecuación a un contexto cultural, a una tradición que determina lo urgente y lo irrelevante, elecciones de diseńo tales como el empleo de ciertos materiales, la figura, o la aplicación del dispositivo a determinadas esferas, y, por último, y acaso más profundamente, los modelos de ser humano que se definen por las diversas formas de concebir los artefactos y sus funciones. Todas estas dimensiones quedan reunidas en tres nociones heideggerianas que se encuentran fuertemente co-implicadas: mundo, situación hermenéutica, y horizonte de comprensibilidad (sentido). 
La noción existencial de mundo lo define como un entramado de relaciones significativas de carácter originariamente pragmático, una red de sentidos interdependientes en la cual cada ente o núcleo significativo dice referencia a los demás, y obtiene de ellos su sentido, cada vez, en función del proyectarse fáctico de una existencia humana. El despliegue de la cotidianidad media encuentra un tipo de ente paradigmático: el útil o artefacto $[$ Zeug]. El uso de artefactos constituye la forma primaria de relación del hombre con el mundo; para esta modalidad, el artefacto se define por su remisionalidad: todo artefacto "es para» $[U m-z u]$ y en esta finalidad, en este quedar referido al fin práctico, radica su esencia. El polo utilitario del ente -su carácter a la mano [Zuhandenheit]- sólo puede ser destacado por una mirada inherente a la ocupación, esta mirada descubridora de la faceta funcional del ente es la denominada Circunspección [Umsicht]. Se trata de una modalidad de la comprensión esencialmente asociada a la «puesta en práctica”, un cierto «saber» habitual y ocupacional.

Ahora bien, dijimos que el mundo se revela primariamente como una red remisional, pues bien, la aproximación que destaca el papel fundamental de la existencia en el circuito de sentido de toda ocupación con útiles convierte a la remisionalidad del artefacto en condición respectiva [Bewandtnis]. En efecto, el entramado de remisiones obtiene su sentido de una serie de referencias interrelacionadas: martillo $\rightarrow$ martillar $\rightarrow$ reparar un tejado $\rightarrow$ evitar filtraciones de agua $\rightarrow$ permitir el habitar del Dasein. Como vemos, existe una remisión final de todas las condiciones respectivas: el denominado por mor de qué [Worumwillen] ${ }^{7}$, y constituye el centro desde donde brota el sentido de la totalidad respeccional [Bewandtnisganzheit]. El hecho de que la serie de condiciones respectivas encuentre su centro y culminación en la existencia humana, se funda en que, por su propia constitución, el Dasein no puede quedar en respectividad de otra cosa, es decir, la existencia no puede convertirse en un medio para. La Worumwillen es la condición de posibilidad para que lo intramundano aparezca como a la mano (Cfr. Tugendhat, 1970:288). El mundo, en tanto entramado de entes y finalidades, articuladas por relaciones de significado que se abren en función del proyectar del Dasein y que lo poseen a él como remisión final, queda caracterizado como Significatividad [Bedeutsamkeit].

\footnotetext{
${ }^{7}$ Claro está, con la expresión Worumwillen, no se mienta únicamente los intereses propios del trato ocupacional con artefactos. También las labores marcadamente menos prácticas como la investigación científica, o la contemplación artística, responden a un por mor de qué, el cual, sin dudas, será capaz de perfilar a los entes según tales o cuales aspectos, aquellos en los que la existencia fija, en cada caso, su interés.
} 
La Apertura [Erschlossenheit] es la estructura unitaria que funciona como condición de posibilidad para toda conexión comprensora del Dasein con el mundo, gracias a ella, la existencia se instala en el mundo y se comporta con respecto al ente. Para el presente estudio, nos interesa particularmente uno de los tres existenciarios que componen la Apertura: la Comprensión [Verstehen] con su posibilidad de articulación, la Interpretación [Auslegung].

El Dasein es definido como un poder-ser, como una posibilidad arrojada [geworfene Möglichkeit] (Cfr. 2006:168) La comprensión nombra el «saber» ${ }^{8}$ inherente a este poder-ser, que se cristaliza en los diversos modos de despliegue de la existencia. En efecto, el ser-posible es siempre transparente para sí mismo en diversos modos y grados. La comprensión es la estructura que permite este «tener noticia» de lo que pasa con la propia existencia. La comprensión es el «entendimiento» (antepredicativo e inexplícito) asociado a todo comportamiento hacia el ser.

La comprensión posee una posibilidad de desarrollo: la interpretación cuya función fundamental es la de apropiarse de lo previamente comprendido. La interpretación toma posesión de lo ya abierto inexplícitamente en el desarrollo de un proyecto del Dasein. La interpretación se realiza desde tres estructuras que cumplen la función de horizonte desde el cual se parcializan los aspectos que incumben a la existencia, "tres presuposiciones que caracterizan la situación hermenéutica» (Kockelmans, 1985:86). Estas estructuras son el haber previo [Vorhabe], la forma previa de ver [Vorsicht] y la forma previa de concebir [Vorgriff]. La Vorhabe nombra la disponibilidad previa de la totalidad respeccional como trasfondo para cualquier modo del comprender; la Vorsicht nombra la orientación previa desde donde se incorpora lo comprendido, un cierto punto de vista que recorta lo pre-comprendido, destacando en ello tales o cuales aspectos; la Vorgriff hace referencia al aparato conceptual, operativo de antemano por medio del cual ocurre toda apropiación interpretativa de lo comprendido. Estos tres momentos constitutivos de la comprensión conforman el sentido [Sinn], es decir, el Horizonte de Comprensibilidad desde donde algo es incorporado por la existencia que comprende.

\footnotetext{
${ }^{8}$ Empleamos las comillas para aclarar que no utilizamos el térmono saber en su genuino sentido de conocimiento tematizante, sino como sinónimo de comprensión en tanto estructura existencial. El saber, asociado al conocimiento que teoriza sobre el mundo, y lo vuelve objeto de su reflexión, encuentra su condición de posibilidad en la estructura ontológica del comprender, es decir, se trata de uno de sus modos.
} 
El horizonte de comprensibilidad ha recibido siempre ya [immer schon] una configuración fáctica e histórica; esto es, un modo particular de modalización de la forma previa de ver, el haber previo, y el modo previo de concebir. Dado que es desde esta configuración histórica -que funciona como trasfondo para la comprensión, y en el que la existencia vive habitualmente- de donde se tomarán los sentidos por medio de los cuales los entes y fenómenos vienen a la presencia, es decir, dado que el horizonte constituye una condición interpretativa del mundo, a este trasfondo se lo conoce como estado interpretativo o situación hermenéutica [hermeneutische Situation]

El estado interpretativo puede configurase de muy diversas maneras, su carácter histórico implica que el mismo se modifica a lo largo de diversas épocas, y para diferentes esferas de la praxis humana. La influencia del estado interpretativo propio de una esfera del quehacer humano puede afectar de manera más o menos profunda el horizonte de comprensión de otra, y, por tanto, el modo de interpretar el mundo y la relación con los demás seres humanos; por ejemplo, la actividad diaria y el modo de abrir el mundo de un zapatero sólo se verá afectado muy transversalmente por los modos dominantes de comprensión que rigen las tareas de la botánica; muy distinta será, desde luego, la situación de un científico dedicado a la investigación del mundo vegetal. Los tres modos previos de la comprensión reciben una disposición específica para cada situación social concreta. El grado de influencia de estos modos previos de comprender dependerán del carácter más o menos abarcativo del fenómeno que ellos mediaticen; entonces, si tenemos en cuenta que, para Heidegger, el modo originario de relación hombre-mundo es la utilización técnica de lo a la mano, comprenderemos que el estado interpretativo asociado al universo del quehacer técnico tendrá una amplia y fundamental influencia en los modos de abrir el mundo, propios de la cotidianidad media.

\section{Una posible articulación}

Hasta este punto hemos descripto sintéticamente la estructura del mundo en la filosofía del primer Heidegger, así como los conceptos de situación hermenéutica y horizonte de comprensibilidad. Consideramos que estas nociones muestran notables semejanzas con el concepto de código técnico de Feenberg, y a continuación, intentaremos hacerlas manifiestas. 
En primer lugar, cabe destacar que la articulación que ahora intentamos es autorizada por el propio Feenberg -indudable conocedor de la filosofía de Heidegger-. En la introducción de su obra de 2002 se afirma expresamente: "las configuraciones técnicas instituyen un "mundo" en un sentido similar al de Heidegger» (Feenberg, 2012:44) Al expresarse de esta manera, Feenberg entiende por mundo «Un marco dentro del cual se generan las prácticas y se ordenan las percepciones» (Feenberg, 2012:44) En efecto, el mundo es en Heidegger una red semántica de sentidos originariamente pragmáticos. Las diferentes formas de estructurarse técnicamente una sociedad proporcionan un entramado de significados desde donde se interpretan los entes y fenómenos, e incluso lo que signifique ser humano.

El mundo funciona como un trasfondo para la comprensión, es decir, abre cooriginariamente una situación hermenéutica o estado interpretativo, en el que la existencia ya se encuentra instalada; una forma previa de comprender al ente, los fenómenos, y las demás existencias humanas. Cada configuración del mundo trae aparejada una comprensibilidad previa, social e históricamente estructurada; esto significa, una forma de definirse el modo previo de ver, haber y concebir (las tres dimensiones previas del sentido).

Cuando Feenberg introduce su noción de código técnico, y la define como un conjunto de regularidades en el diseño y trasfondo para las decisiones técnicas, la resonancia de los conceptos de Heidegger se hace perceptible. El canadiense indica en el capítulo 3 de su obra de 2002 que la noción de código puede significar dos cosas, a saber, a) una regla que clasifica actividades como permitidas o prohibidas, y b) una regla que asocia esas reglas a un significado o propósito (Feenberg, 2012:126). El primer significado de código nos recuerda a la noción heideggeriana de vorsicht, esto es, la forma previa de ver, tal como queda definida de antemano por un horizonte histórico de comprensibilidad. El modo previo de ver determina de antemano, como qué un ente o fenómeno se hará presente ante la existencia ocupada. En el caso de la configuración técnica de la sociedad, esta visibilidad previa, o «modo previo de problematizar» las cuestiones tecnológicas inherente al código técnico es la que determina lo que puede o no hacerse; por ejemplo, el desarrollo o de armas de guerra que generan «sufrimientos innecesarios»" ${ }^{9}$ o que producen "fragmentos no localizables por rayos

\footnotetext{
${ }^{9}$ Convención sobre prohibiciones o restricciones del empleo de ciertas armas convencionales que puedan considerarse excesivamente nocivas o de efectos indiscriminados, textos de la convención y de los protocolos aprobados hasta el 28 de noviembre de 2003, comité internacional de la
} 
$\mathrm{X}{ }^{10}$, ha sido prohibido por protocolos internacionales ${ }^{11}$. El modo previo de ver o problematizar también determina lo relevante o irrelevante para un sistema técnico históricamente contextualizado, por ejemplo, el desarrollo de fuentes de energías renovables y no contaminantes no era una prioridad en los ańos '30, pero está llegando a serlo en nuestros días; asimismo, el diseño de abrochadoras más pequeñas, adaptadas a las manos de las mujeres no era un problema en los años '60, pero pasó a serlo durante la década del '70, con el ingreso de un significativo número de mujeres a puestos de trabajo antes reservados únicamente para hombres. (Cfr. Con el estudio de Hoag, 1982:254).

El segundo significado de código, se asemeja al concepto heideggeriano de Vorgriff, o modo previo de concebir. Esta dimensión de la precomprensión asocia la decisión de diseño o aplicación a una conceptualidad previa. En el caso de las sociedades actuales, técnicamente estructuradas en torno al modelo capitalista, las elecciones y aplicaciones de dispositivos se realizan desde nociones como las de eficiencia y productividad; lentamente comienzan a hacerse presentes otros conceptos que mediatizan la decisión, como las categorías de contaminante o insalubre, o bien, la contraposición renovable/no-renovable. Estas nociones -y todas las que definen un modo previo de conceptualizar- son categorías que entran y salen de vigencia. Según Feenberg, las tecnologías encierran potencialidades, tanto de dominación como de liberación; pero para que estas potencialidades sean efectivizadas -en uno u otro sentido-, es necesaria la modificación del código técnico, es decir, el horizonte de comprensibilidad de la dimensión técnico-histórica de una sociedad.

La posibilidad de un cambio civilizatorio está latente en las máquinas de las que hoy disponemos. La tecnología no es un destino irrevocable, como pretendía la posición sustantiva. Un cambio en la conceptualidad previa que mediatiza a interpretación de los entes y fenómenos es el camino para la modificación de la escena técnica, una variación que llevaría a destacar nociones que conduzcan a una disposición menos represiva de las tecnologías.

En este sentido indica Feenberg que las potencialidades de la tecnología son vistas como suboptimizaciones por la cultura económica dominante, pero, si el

cruz roja, ginebra, 2006, Protocolo II "sobre prohibiciones o restricciones del empleo de minas, armas trampa y otros artefactos", p.28

${ }^{10}$ Ibid., Protocolo I "Sobre fragmentos no localizables", p.23

${ }^{11}$ La efectividad de esta prohibición es otra cuestión. 
código técnico es modificado, este modo de interpretar sería consecuentemente reemplazado; en efecto, una caída en la productividad no sería vista como un efecto nocivo en una sociedad para la cual el bienestar del trabajador, o la protección del medio ambiente fuera un valor fuerte, un modo previo de concebir. Si_se incluyesen nociones diferentes (las así llamadas «variables blandas», tales como seguridad ocupacional, o aire limpio) en el código técnico, una caída en la producción no sería interpretada como un inconveniente, en el caso de que sirviese, por ejemplo, al bienestar laboral (Cfr. Feenberg, 2012:224)

La teoría de Feenberg posee otro punto de contacto con la filosofía del primer Heidegger, a saber, en lo referente a la discusión acerca del papel determinante de la organización técnica del mundo en la configuración de los caracteres estructurales de la existencia humana.

Como pudimos ver, para Feenberg, lo que significa ser humano se decide en la forma de las herramientas. Consideramos, no sólo que esta noción ya se encuentra presente en la filosofía del primer Heidegger, sino que se trata de una noción central en su filosofía de la praxis.

En nuestra opinión, Heidegger estaría de acuerdo con la consideración de Feenberg, pero tal vez acentuaría el carácter recíproco de la relación existencia-herramienta. La Analítica existencial del Dasein se encuentra pronto con la noción de mundo, éste se vuelve un concepto central y que acompaña todo el desarrollo del proyecto del primer período de la filosofía del autor alemán. La modalidad del mundo con la que la existencia se relaciona inmediata y regularmente es la de mundo circundante [Umwelt], esta noción hace referencia a aquello que inmediatamente nos compete en tanto nos relacionamos originariamente con el entorno. El despliegue originario de la existencia es ocupacional y el mundo descubierto por esta forma de proyectarse no es otra cosa que el mundo estructurado técnicamente, el mundo de la ocupación, y de las conexiones remisionales de los útiles, el «mundo del trabajo» [Werkwelt] (Cfr. Heidegger, 2007a: $\$ 23 \mathrm{~b}-\alpha)$ o en otras palabras, el «mundo de las configuraciones técnicas» resaltado por Feenberg (Cfr. Feenberg, 2012:44). Las posturas de Heidegger y Feenberg coinciden en la consideración de que que el ente técnico nunca es algo «en sí» y con una esencia invariable y desligada del contexto (Feenberg 2012:80), sino siempre imbricado en un mundo, y relacionado con una existencia, de donde obtiene su sentido. Este carácter vivencial e históricamente contextualizado del fenómeno técnico es justamente lo que queda destacado por medio de la introducción del 
concepto de código técnico: el fenómeno técnico es multifacético, e involucra dimensiones ajenas a la mera efectividad o productividad.

La multidimensionalidad de la situación tecnológica ${ }^{12}$ exhibe un carácter adicional, que es destacado por ambos autores, a saber, su operatividad desde un trasfondo, o bien, su inmediata inexplicitud. A esto se refiere Feenberg cuando presenta el concepto de «inconsciente tecnológico» (Feenberg, 2012:48), que no sería otra cosa que el código técnico en tanto que opera desde su carácter nomanifiesto; lo mismo que ocurre con la situación hermenéutica, por vía de la definición de los tres niveles de la precomprensión.

El papel decisivo de la existencia humana en la configuración del mundo técnico (y la correlativa modalización de la existencia por su instalación en este contexto pragmático) aparece condensada en la noción heideggeriana de Worumwillen, o por mor de qué (Rivera). Este concepto nombra el punto culminante en el cual desembocan todas las remisiones del mundo técnico. En efecto, los para qué de los artefactos se encadenan en una secuencia de finalidades que concluyen en un central para quien, de donde emana el sentido de su concatenación; a su vez, este para quien no puede volverse condición respectiva de otra cosa.

Desde esta perspectiva, la técnica dista de ser un destino irrevocable, tal como lo pretendía la posición sustantiva que describe Feenberg. Ambos autores coinciden en la consideración de que no hay algo así como un ser humano a-técnico al cual la técnica dé forma, y tampoco hay una técnica neutral, no-humana a la cual una existencia dé sentido, finalidad y valor. La técnica y la existencia se influyen recíprocamente. Las consideraciones de Heidegger hacen eco en expresiones de Feenberg tales como estas dos, que pueden leerse en correlación la una con la otra: «seguir de manera persistente uno u otro camino técnico define al usuario como un tipo humano u otro” (Feenberg, 2012:39) Y esta otra: «En realidad no existe algo tal como la tecnología "en sí misma", dado que las tecnologías sólo existen en el contexto de un modo u otro de uso» (Feenberg, 2012:80) La relación recíproca entre la técnica y la existencia se vuelve manifiesta en la filosofía de ambos autores.

Pero podríamos extender aún más la familiaridad entre ambas teorías: en la introducción de su obra de 2002, Feenberg expone el siguiente interrogante: «la pregunta más importante para hacerse en relación con las sociedades modernas es

\footnotetext{
${ }^{12}$ Nos permitimos utilizar esta expresión para indicar que el modo previo de configurarse el mundo de la praxis es una forma de configuración de la Situación hermenéutica.
} 
¿qué significado de la vida humana está contenido en las configuraciones técnicas dominantes?» (Feenberg, 2012:44) Este cuestionamiento se retoma en el capítulo 7: «[una teoría de la instrumentalización secundaria] nos dirá qué pasa con las personas cuyas vidas están dedicadas a trabajar la madera, cómo esa actividad dará forma a sus manos, sus reflejos, su lenguaje y su personalidad, de modo tal que tenga sentido llamar carpintero a alguien» (Feenberg, 2012:273). Las preguntas de Feenberg se dirigen a la constitución del sentido de ser humano por su interrelación con el universo técnico. Este tipo de cuestionamiento, que se aleja de la pregunta por el fenómeno "objetiva y teoréticamente aislado», y se dirige a sus condiciones de posibilidad, que arraigan en la existencia humana, es un típico interrogante existencial heideggeriano. Podemos observar un ejemplo de esta forma de preguntar en este fragmento del parágrafo 69b de Ser y Tiempo: «¿Cuáles son, en la constitución de ser del Dasein las condiciones de posibilidad existenciales necesarias para que el Dasein pueda existir en la forma de la investigación científica?» (Heidegger, 2006:373). El mismo interrogante podría dirigirse al quehacer técnico en tanto posibilidad de comportamiento del Dasein. Vemos que la pregunta no es por el fenómeno desarraigado, sino por cómo es esa existencia que se comporta científicamente, o en el caso que nos compete, técnicamente.

El viraje del preguntar hacia la existencia humana como centro de emanación del sentido y el correlativo interés por la configuración de esta existencia al comportarse técnicamente es característica de un preguntar Analítico-existencial. Consideramos que puede encontrarse en Heidegger una radicalización del planteo de Feenberg, una base ontológica muy pertinente (una teoría del existir humanotécnico) que puede convertirse nada menos que en el fundamento para esa teoría de la instrumentalidad secundaria que Feenberg solicita.

\section{Conclusiones}

A lo largo de las páginas precedentes pudieron observarse una serie de puntos de contacto entre la teoría crítica de la tecnología de Feenberg y la filosofía existencial de la praxis de Heidegger. Específicamente, encontramos fuertes paralelismos en las siguientes cuestiones:

a) Las nociones de mundo, situación hermenéutica y horiznte de comprensibilidad constituyen un importante antecedente para el concepto de código técnico introducido por Feenberg. Los fenómenos expuestos por Heidegger 
coinciden en describir el trasfondo, histórica y socialmente constituido y operante de antemano desde el cual se determina el sentido de los entes con los que la existencia humana se relaciona. Específicamente, la noción de mundo daría cuenta del carácter reticular y semántico del ámbito pragmático en el que el ser humano se moviliza desde siempre; el horizonte de comprensibilidad destacaría el carácter predefinido e inexplícito de las decisiones de diseño, pero también de cuestiones como la determinación de lo urgente, lo irrelevante, la conceptualidad por medio de la cual se comprende lo producido, la materia de la que se construye el artefacto, el modo de problematizar las cuestiones técnicas, y lo que signifique ser humano en función del quehacer práctico. Por último, la situación hermenéutica hace referencia al carácter histórico y socialmente situado de un horizonte de comprensibilidad, así como su estructura variable y contextual. Todas estas concepciones aparecen recogidas de manera sintética en el concepto de código técnico.

b) Feenberg declara la necesidad de elaborar una teoría de la instrumentalidad secundaria, que consistiría en la investigación de la forma en que el código técnico interseca con el artefacto produciendo una configuración fáctica específica en este último, pero también, y acaso fundamentalmente, en las decisiones de diseño y en la construcción de la subjetividad de un tipo de usuario concreto. Una teoría de la instrumentalidad secundaria explicaría cómo la herramienta se vuelve a la vez condición para el proyectar de la existencia humana y resultado de este desarrollo fáctico, siempre socialmente situado. En nuestro estudio procuramos mostrar que la filosofía existencial de la praxis del primer Heidegger ofrece muy pertinentes bases para ejercitar la reconducción de la forma concreta de la herramienta hacia los modos del ser-en-el-mundo que operan como su trasfondo -lo que Winograd y Flores denominan «diseño ontológico» (Cfr. Flores y Winograd, 1986: 179)-. También señalamos que el concepto de worumwillen coloca a la existencia humana en el centro de confluencia de todas las condiciones respectivas constitutivas del mundo circundante; esta perspectiva avanza en la misma dirección que el trabajo propuesto por Feenberg, a saber, la elaboración de un discurso acerca de cómo el ser humano queda implicado en sus herramientas y es modificado por ellas, al tiempo que varía su misma relación con el mundo y los otros. Sugerimos que la filosofía de la praxis del primer Heidegger puede ser interpretada como la realización previa del proyecto de teoría de la instrumentalización secundaria solicitado por Feenberg. 
c) Por último, consideramos que el motivo por el cual, en la división inicial de interpretaciones acerca de la técnica realizada por Feenberg ${ }^{13}$, Heidegger es presentado como representante de la posición sustantiva, es que Feenberg centra su escrito casi exclusivamente en las obras del Heidegger posterior a la Kehre de los años '30. En efecto, encontramos en los textos del segundo Heidegger afirmaciones visiblemente negativas acerca de la técnica, que hacen de ella el modo eminente del pensar contemporáneo y el último capítulo en la historia del olvido del ser, es decir, el eslabón culminante del pensar metafísico, tendiente a la dominación de la naturaleza (Heidegger, 2001:15, por poner un ejemplo). Estas interpretaciones explican la -algo exagerada- descripción de Heidegger como un autor «fatalista» (Feenberg, 2012:28). Feenberg afirma: «Heidegger y Ellul confunden la esencia de la tecnología con el código hegemónico actual» (Feenberg, 2012:135). Consideramos que esta expresión revela la selección de un determinado terreno de estudio; esta afirmación no podría realizarse tan fácilmente si la atención del autor se hubiese detenido en las obras anteriores a los años '30. Sin dudas, los estudios del segundo período del pensar de Heidegger presuponen y se basan en los del primero, pero la aproximación más tardía no expande los resultados de los escritos tempranos, sino que lleva los análisis hacia otros horizontes. En el segundo Heidegger no encontramos una reflexión pormenorizada acerca del modo de existencia del objeto técnico, más bien, estos objetos se convierten en puntos de condensación de un modo general del pensar, que propicia la provocación de la naturaleza. Esta reflexión surge en el marco de un proyecto amplificado de examinación de la manera en que el ser comparece ante el modo calculador de aproximarse a los entes. En contraste, las reflexiones del primer Heidegger observan al artefacto desde una cierta neutralidad valorativa. Si bien la temática está visiblemente presente en ambos períodos, puede observarse que no hay -antes y después de la Kehre de los '30- una creciente especificación, sino más bien una reconducción de la problemática. En las obras heideggerianas de los años '20 encontramos una completa y profunda filosofía de la praxis, en tanto que arraigada en el mundo técnico, la cual presenta al ser humano (modificado y modificante) en su centro. Sugerimos que, de haberse centrado en las obras del primer período del pensar heideggeriano (específicamente, el período de Marburgo 1923-1927) Feenberg no hubiese presentado en su escrito a Heidegger como un representante de la teoría sustantiva, por el contrario, hubiese encontrado un valioso antecedente y fundamento para su propia teoría crítica de la tecnología.

${ }^{13}$ Teoría instrumental, teoría sustantiva, y teoría crítica 


\section{Bibliografía}

Bud, R. y WARnER, D (Eds.)

-(1998) Instruments of science, an historical encyclopedia, Garland Publishing Inc.: London.

Brandom, R.

-(2002a) "Heidegger's Categories in Sein und Zeit" en Tales of the Mighty dead: Historical Essays in the Metaphysics of Intentionality, Harvard University Press, Cambridge, Massachusetts/ London, England.

-(2002b) "Dasein, the Being that Thematizes" en Tales of the Mighty dead: Historical Essays in the Metaphysics of Intentionality, Harvard University Press, Cambridge, Massachusetts/ London, England.

Feenberg, A.

-(2002) Transformar la tecnología, una nueva visita a la teoría crítica. Ed. Universidad Nacional de Quilmes, Buenos Aires, Argentina.

-(1991) Critical Theory of Technology, Reino Unido, Oxford University Press.

-(1995) Alternative Modernity, Estados Unidos, University of California Press.

-(1999) Questioning Technology, USA y Canada, Routledge.

-(2002) Transforming Technology: A Critical Theory Revisited, Reino Unido, Oxford University Press.

-(2005) Heidegger and Marcuse: The Catastrophe and Redemption of History, USA y Canada Routledge.

GilliLAND, R.:

-(2002) "The destiny of technology : Modern science and human freedom in the later Heidegger", en: Heidegger Studien / Heidegger studies / Etudes heidegeriennes No18, pp.115-128.

Heidegger, M:

-(1994) La pregunta por la técnica, en Conferencias y artículos, Barcelona: Ediciones de Serbal, Traducción de Eustaquio Barjau.

-(2000): Los problemas fundamentales de la fenomenología, Madrid:Trotta, Trad. y prólogo de Juan José García Norro.

-(2002): Interpretaciones fenomenológicas sobre Aristóteles, indicación de la situación hermenéutica [informe Natorp] Madrid: Trotta. Trad. de Jesús Adrián Escudero.

-(2003) Plato's Sophist, Indiana, USA: Indiana University Press. Trad. De Richard Rojcewicz y André Schuwer. 
-(2004): Lógica: la pregunta por la verdad, Madrid: Alianza. Versión española de J. Alberto Ciria.

-(2006) Ser y Tiempo, Madrid: Trotta. Traducción y notas de Jorge Eduardo Rivera.

-(2007) Seminarios de Zollikon: protocolos, diálogos, cartas. México: Jitanjáfora Mo Relia Ed. Trad. De Ángel Xolocotzi Yáñez.

HoAg, L.

-(1982) Anthropometric and Strength Data in Tool Design. En Easterby R. Eds. Anthropometry and Biomechanics: Theory and Application. Plenum press, New York. pp.253-257.

Kockelmans, J.J.

-(1985): Heidegger and Science, Washington D.C.: Center for advanced research in phenomenology \& University Press of America.

PARENTE, D.

-(2008a): "La concepción heideggeriana del Artefacto en Grundbegriffe der Metaphysik" en Signos Filosóficos, vol. X, núm. 20, julio-diciembre, pp. 75-93.

(2008b) "Observaciones sobre uso y función de artefactos en Sein und Zeit de M. Heidegger” en Contrastes, Revista internacional de filosofía, Vol.XIII, Málaga, España: Ed. De la Universidad de Málaga, pp. 37-59.

Tugendhat, E.

-(1970). Der Wahrheitsbegriff bei Husserl und Heidegger, Berlin: Walter de Gruyter \& Co.

Winograd, T., \& Flores, F.

-(1986). Understanding Computers and Cognition, California: Addison-Wesley.

Enviado: 4/08/2016

Aceptado: 16/11/2016

\section{(9) $(0 \Theta \Theta$}

ENDOXA está bajo una licencia de Creative Commons Reconocimiento-NoComercial-SinObraDerivada 4.0 Internacional 
